\title{
Prevention of Evisceration or Enucleation in a Patient with Endogenous Klebsiella Panophthalmitis and Scleral Abscess
}

\author{
Kuan-Jen Chen* \\ Department of Ophthalmology, Chang Gung Memorial Hospital and College of Medicine, Chang Gung University, Taoyuan,, Taiwan
}

A 70-year-old diabetic man with Klebsiella liver abscess complained of painful blurred vision in the right eye for 7 days and was referred for evisceration or enucleation. Visual acuity was no light perception. Slit-lamp examination showed superotemporal scleral abscess, corneal ring-shaped infiltration and mild ocular deformity (Figure 1A). After three consecutively intravitreal and periocular injections of ceftazidime/dexamethasone combinations within one week, the abscess subsided without deformity of the eyeball (Figure 1B). Additionally, he received five consecutively intravitreal injections, the infection and inflammation subsided without recurrences during 1-year follow-up.

Severe panophthalmitis is frequently accompanied by corneal opacity, scleral abscess, and perforation or rupture. In previous reports, enucleation or evisceration was the only remaining viable treatment option when all options to salvage the eye had been exhausted. This case showed endogenous bacterial panophthalmitis and scleral abscess could be controlled by multiple intravitreal and periocular injections of antibiotics and dexamethasone.

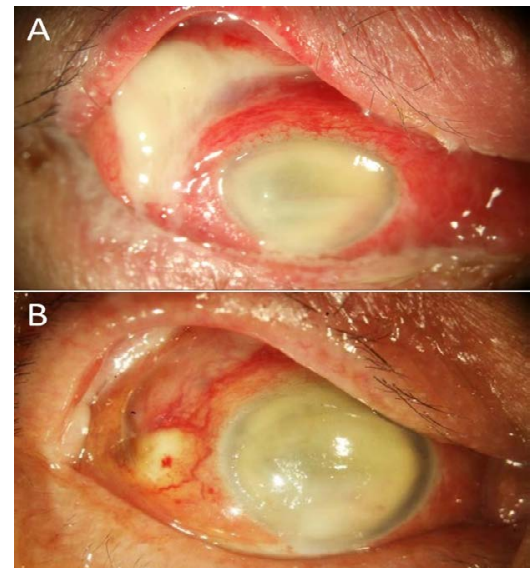

Figure 1. Endogenous Klebsiella Panophthalmitis and Scleral Abscess. A, slit-lamp examination showed superotemporal scleral abscess, corneal ring-shaped infiltration and mild ocular deformity. B, The abscess subsided without deformity of the eyeball after three consecutively intravitreal and periocular injections of ceftazidime/dexamethasone combinations
Copyright: (C2018 Chen K. This is an open-access article distributed under the terms of the Creative Commons Attribution License, which permits unrestricted use, distribution, and reproduction in any medium, provided the original author and source are credited.
${ }^{\star}$ Correspondence to: Kuan-Jen Chen, Department of Ophthalmology, Chang Gung Memorial Hospital, 5 Fuhsing Street, Kwei-Shan, 333, Taoyuan, Taiwan, Tel: 886-3-3281200 ext. 8671; Fax: 886-3-328-7798; E-mail: cgr999chiayi@ yahoo.com.tw

Received: October 01, 2018; Accepted: October 11, 2018; Published: October 15,2018 